\title{
APPROXIMATE POINT SPECTRUM OF A WEIGHTED SHIFT
}

\author{
BY \\ WILLIAM C. RIDGE
}

Notation. If $T$ is a Hilbert space operator, let $\Lambda(T)$ denote its spectrum, $\Pi(T)$ its approximate point spectrum, $\Pi_{0}(T)$ its point spectrum, $\Gamma(T)$ its compression spectrum, $m(T)$ its lower bound (i.e., inf $\{\|T x\| /\|x\|: x \neq 0\}$ ), and $r(T)$ its spectral radius. Let $i(T)$ denote $\sup _{n} m\left(T^{n}\right)^{1 / n}$, which equals $\lim _{n} m\left(T^{n}\right)^{1 / n}$.

Let $R$ denote a weighted right shift on $l_{+}^{2}$, defined by $R e_{n}=s_{n} e_{n+1}$, where $\left(e_{n}\right)$ is an orthonormal basis of $l_{+}^{2}, n=1,2, \ldots$ Let $L$ denote its adjoint, a weighted left shift. Let $B$ denote a weighted two-sided shift on $l^{2}$, defined by $B e_{n}=s_{n} e_{n+1}$, $n=0, \pm 1, \pm 2, \ldots,\left(e_{n}\right)$ here being an orthonormal basis of $l^{2}$. If $B$ has purely nonzero weights $\left(s_{n}\right)$, then let

$$
\begin{array}{ll}
i(B)^{-}=\lim _{n} \inf _{k \leqq 0}\left|s_{k-1} \cdots s_{k-n}\right|^{1 / n}, & i(B)^{+}=\lim _{n} \inf _{k \geqq-1}\left|s_{k+1} \cdots s_{k+n}\right|^{1 / n}, \\
r(B)^{-}=\lim _{n} \sup _{k \leqq 0}\left|s_{k-1} \cdots s_{k-n}\right|^{1 / n}, & r(B)^{+}=\lim _{n} \sup _{k \geqq-1}\left|s_{k+1} \cdots s_{k+n}\right|^{1 / n} .
\end{array}
$$

Background. $\Lambda$ and its parts, for weighted shifts, are known to have circular symmetry about $0 ; \Pi_{0}(R)$ is known to be empty or $\{0\} ; \Gamma(R)$ is known to be a disk, possibly degenerating to $\{0\}$; and $\Gamma(B)$ and $\Pi_{0}(B)$ are known to be annuli, possibly degenerate or empty, and in any case disjoint. These facts are easy to verify, and are proved in [3]. Also proved there is the much deeper fact that the spectrum of a weighted shift is always connected. This will also be deduced in this paper as an easy corollary of the results on the approximate point spectrum. Some of these results, from a different approach, seem to have been concurrently proved in [1].

The following formulae are easy to verify [3]:

$$
i(R)=\lim _{n} \inf _{k}\left|s_{k+1} \cdots s_{k+n}\right|^{1 / n}, \quad r(R)=\lim _{n} \sup _{k}\left|s_{k+1} \cdots s_{k+n}\right|^{1 / n} .
$$

Preliminaries. The following propositions can be verified by routine arguments [4].

(1) Suppose (for $R$ ) that no $s_{n}$ vanishes and $\varepsilon, M$ are positive numbers. Then there are integers $k$ and $n$, both greater than $M$, such that $\left|s_{k+1} \cdots s_{k+n}\right|^{1 / n}>r(R)-\varepsilon$.

(2) For any positive numbers $A, B, C, D$,

$$
\frac{A+B}{C+D} \leqq \text { maximum }\left(\frac{A}{C}, \frac{B}{D}\right) \text {. }
$$

Received by the editors April 22, 1969. 
(3) Suppose $\left(p_{n}\right)$ is a real nonnegative periodic sequence having period $r$, and $q=\left(p_{1} \cdots p_{r}\right)^{1 / r}$. Suppose $\left(a_{n}\right)$ is a real nonnegative sequence such that $\lim _{n}\left(a_{n}-p_{n}\right)$ $=0$. If either some $p_{n}$ vanishes, or no $a_{n}$ vanishes, then

$$
\lim _{n} \inf _{k}\left(a_{k+1} \cdots a_{k+n}\right)^{1 / n}=\lim _{n} \sup _{k}\left(a_{k+1} \cdots a_{k+n}\right)^{1 / n}=q .
$$

(4) Suppose (for $B$ ) that no $s_{n}$ vanishes. Then $i(B)=$ minimum $\left(i(B)^{-}, i(B)^{+}\right)$ and $r(B)=$ maximum $\left(r(B)^{-}, r(B)^{+}\right)$.

(5) For any operator $T, \Pi(T) \subset\{c: i(T) \leqq|c| \leqq r(T)\}$.

THEOREM 1. If no $s_{n}$ vanishes, then $\Pi(R)=\{c: i(R) \leqq|c| \leqq r(R)\}$. If finitely many $s_{n}$ vanish, then $\Pi(R)=\{0\} \cup \Pi\left(R^{\prime}\right)$, where $R^{\prime}$ is the right shift with weights $s_{k+1}$, $s_{k+2}, \ldots$, where $s_{k}$ is the last zero weight. If infinitely many $s_{n}$ vanish, then

$$
\Pi(R)=\{c:|c| \leqq r(R)\} .
$$

Proof. If $i(R)=r(R)$, then $\Pi(R)$ is by (5) contained in, hence by nonemptiness and circular symmetry equal to, $\{c:|c|=r(R)\}$.

Suppose no $s_{n}$ vanishes, and $i(R)<c<r(R)$. Since $\Pi(R)$ is closed and has circular symmetry, it suffices in view of (5), for the first assertion of the theorem, to show that $c$ is necessarily in $\Pi(R)$.

Choose numbers $a, b$ such that $i(R)<a<c<b<r(R)$, and suppose $\varepsilon>0$. Choose $n$ such that $(c / b)^{n}<\varepsilon$ and $k$ such that $\left|s_{k+1} \cdots s_{k+n}\right|^{1 / n}>b$. By (1) choose $p$ such that $(a / c)^{p}<\varepsilon$ and $m$ such that $m>n+k$ and $\left|s_{m+1} \cdots s_{m+p}\right|^{1 / p}<a$.

Define $x=\left(x_{i}\right)$ by

$$
\begin{array}{rlrl}
x_{k+1} & =1, & \\
x_{r} & =\frac{s_{k+1} \cdots s_{r-1}}{c^{r-k-1}} & & \text { if } k+2 \leqq r \leqq m+p+1, \\
x_{r} & =0 & \text { if } r<k+1 \text { or } r>m+p+1 .
\end{array}
$$

Then

and hence

$$
\begin{aligned}
R x-c x & =\sum_{r=k+1}^{m+n+1}\left(\frac{s_{k+1} \cdots s_{r}}{c^{r-k-1}} e_{r+1}-\frac{s_{k+1} \cdots s_{r-1}}{c^{r-k}} e_{r}\right) \\
& =s_{m+p+1} x_{m+p+1} e_{m+p+2}-c e_{k+1}
\end{aligned}
$$

Also

$$
\begin{aligned}
\|R x-c x\|^{2} & =\left|s_{m+p+1}\right|^{2}\left|x_{m+p+1}\right|^{2}+c^{2} \\
& \leqq\|R\|^{2}\left(1+\left|x_{m+p+1}\right|^{2}\right) .
\end{aligned}
$$

But

and

$$
\|x\|^{2}=\sum\left|x_{i}\right|^{2} \geqq\left|x_{k+n+1}\right|^{2}+\left|x_{m+1}\right|^{2} .
$$

$$
\left|x_{k+n+1}\right|=\left|s_{k+1} \cdots s_{k+n}\right| / c^{n}>(b / c)^{n}>1 / \varepsilon
$$

$$
\left|x_{m+p+1}\right| x_{m+1}|=| s_{m+1} \cdots s_{m+p} \mid / c^{n}<(a / c)^{p}<\varepsilon .
$$


So by (2),

and so $c$ is in $\Pi(R)$.

$$
\begin{aligned}
\frac{\|R x-c x\|^{2}}{\|x\|^{2}} & \leqq\|R\|^{2} \frac{1+\left|x_{m+p+1}\right|^{2}}{\left|x_{k+n+1}\right|^{2}+\left|x_{m+1}\right|^{2}} \\
& \leqq\|R\|^{2} \max \left(\frac{1}{\left|x_{k+n+1}\right|^{2}},\left|\frac{x_{m+p+1}}{x_{m+1}}\right|^{2}\right) \\
& <\varepsilon^{2}\|R\|^{2}
\end{aligned}
$$

Now suppose infinitely many $s_{n}$ vanish, and suppose $0<c<r(R)$. The last assertion of the theorem will follow if we show that, necessarily, $c$ is in $\Pi(R)$.

Choose $b$ such that $c<b<r(R)$. Given $\varepsilon>0$, choose $n$ such that $(c / b)^{n}<\varepsilon$ and $k$ such that $\left|s_{k+1} \cdots s_{k+n}\right|^{1 / n}>b$. Let $r$ be the first index greater than $k+n$ such that $s_{r}=0$. Define $x=\left(x_{i}\right)$ by

$$
\begin{aligned}
x_{k+1}=1, & \\
x_{m}=\frac{s_{k+1} \cdots s_{m-1}}{c^{m-k-1}} & \text { if } k+2 \leqq m \leqq r, \\
x_{m}=0 & \text { if } m \leqq k \text { or } m>r .
\end{aligned}
$$

Then $R x-c x=c e_{k+1},\|R x-c x\|=c$, and

$$
\|x\| \geqq\left|x_{k+n+1}\right|=\left|s_{k+1} \cdots s_{k+n}\right| / c^{n}>(b / c)^{n}>1 / \varepsilon
$$

so $\|R x-c x\| /\|x\|<c \varepsilon$, and $c$ is in $\Pi(R)$.

If finitely many $s_{n}$ vanish, then $R$ is the orthogonal sum of $R^{\prime}$ and a nilpotent operator, and $\Pi(R)=\Pi\left(R^{\prime}\right) \cup\{0\}$; applying the earlier argument for nonzero weights to $R^{\prime}$, we have the second assertion.

Corollary (Kelley). $\Lambda(R)=\{c:|c| \leqq r(R)\}$.

Proof. $\Pi$ contains the boundary of $\Lambda$, which is therefore either the annulus $\Pi$ or the closed disk of radius $r(R)$; it is the latter since 0 is in $\Gamma(R)$.

Definition. A sequence $\left(a_{n}\right)$ is almost periodic if there is a periodic sequence $\left(p_{n}\right)$ such that $\lim _{n}\left(a_{n}-p_{n}\right)=0$. If $r$ is the period of $\left(p_{n}\right)$, the periodic mean is $\left(p_{1} \cdots p_{r}\right)^{1 / r}$.

THEOREM 2. If $\left(\left|s_{n}\right|\right)$ is almost periodic, then $\Pi(R)=\{c:|c|=q\}$ if all $s_{n}$ are nonzero, and is the same set together with $\{0\}$ if some $s_{n}$ vanishes; in either case $\Lambda(R)=$ $\{c:|c| \leqq q\}$, where $q$ is the periodic mean of the approximating periodic sequence $\left(p_{n}\right)$.

Proof. The last statement follows from the first two by the corollary to Theorem 1.

If either some $p_{n}$ vanishes or no $s_{n}$ vanishes, then $i(R)=r(R)=q$ by (3), and $\Pi(R)$ is as asserted by Theorem 1 .

Suppose no $p_{n}$ vanishes, but some $s_{n}$ vanishes. Then only finitely many $s_{n}$ vanish, since $\lim _{n}\left(\left|s_{n}\right|-p_{n}\right)=0$ and $\left(p_{n}\right)$, assuming only finitely many distinct values, is bounded away from 0 . Theorem 1 now applies again, and $\Pi(R)$ is as asserted. 
Corollary 1. If $R$ is injective and $\left|s_{n}\right| \rightarrow s$, then

$$
\Pi(R)=\{c:|c|=s\} \text { and } \Lambda(R)=\{c:|c| \leqq s\} .
$$

COROLlaRY 2. If $\left(\left|s_{n}\right|\right)$ is periodic with mean $q$, then

$$
\Pi(R)=\{c:|c|=q\} \text { and } \Lambda(R)=\{c:|c| \leqq q\} .
$$

ExAmple. Let $T=$ subdiagonal $(1,2,1,2, \ldots)$. By Corollary 2 ,

$$
\Pi(T)=\{c:|c|=\sqrt{ } 2\} \text { and } \Lambda(T)=\{c:|c| \leqq \sqrt{ } 2\} .
$$

Note. If some $s_{n}=0$ for $B$, then $B$ is the orthogonal sum of a right and a left shift, and their approximate point spectra are described elsewhere in this paper. In treating $B$ below, we therefore assume that no $s_{n}$ vanishes.

THEOREM 3. If $r(B)^{-}<i(B)^{+}$, then

$$
\Pi(B)=\left\{c: i(B)^{+} \leqq|c| \leqq r(B)^{+} \text {or } i(B)^{-} \leqq|c| \leqq r(B)^{-}\right\} .
$$

Otherwise $\Pi(B)=\{c: i(B) \leqq|c| \leqq r(B)\}$.

Proof. Since $\Pi(B)$ is closed and has circular symmetry, in view of (4) and (5) we need only consider positive $c$ lying between any two of the values $i(B)^{-}, i(B)^{+}$, $r(B)^{-}$and $r(B)^{+}$.

If $i(B)^{+}<c<r(B)^{+}$, then exact imitation of the construction of Theorem 1 (with $i(B)^{+}<a<c<b<r(B)^{+}$) yields approximate eigenvectors for $c$. So

$$
\left\{c: i(B)^{+} \leqq|c| \leqq r(B)^{+}\right\} \subset \Pi(B) .
$$

Suppose $i(B)^{-}<c<r(B)^{-}$. Choose numbers $a, b$ such that $i(B)^{-}<a<c<b$ $<r(B)^{-}$. Choose $p$ such that $(a / c)^{p}<\varepsilon$ and $m<-p$ such that $\left|s_{m+1} \cdots s_{m+p}\right|^{1 / p}<a$. Choose $n$ such that $(c / b)^{n}<\varepsilon$ and, by (1), choose $k<m-n$ such that $\left|s_{k+1} \cdots s_{k+n}\right|^{1 / n}$ $>b$. Define $x$ as in the proof of Theorem 1, and again we find $c \in \Pi(B)$. So

$$
\left\{c: i(B)^{-} \leqq|c| \leqq r(B)^{-}\right\} \subset \Pi(B) \text {. }
$$

Suppose $r(B)^{+}<c<i(B)^{-}$. Choose $a, b, n, p$ as before; choose $k<-n$ such that $\left|s_{k+1} \cdots s_{k+n}\right|^{1 / n}>b$, and $m \geqq 0$ such that $\left|s_{m+1} \cdots s_{m+p}\right|^{1 / p}<a$. Proceeding as in the proof of Theorem 1, we find that $c$ is in $\Pi(B)$. So if $r(B)^{+} \leqq i(B)^{-}$, then

is contained in $\Pi(B)$.

$$
\left\{c: r(B)^{+} \leqq|c| \leqq i(B)^{-}\right\}
$$

Suppose $r(B)^{-}<c<i(B)^{+}$. We show that $c$ is not in $\Pi(B)$. Suppose it were. Choose $b$ strictly between $c$ and $i(B)^{+}$. Then for some $N$ we have, for all $n \geqq N$ and $k \geqq 1,\left|s_{k+1} \cdots s_{k+n}\right|^{1 / n}>b$. For all positive $\varepsilon$, choose a unit vector $x=x(\varepsilon)$ such that $\|B x-c x\|<\varepsilon$.

Suppose there exists a sequence $\varepsilon^{\prime} \rightarrow 0$ such that $x_{0}\left(\varepsilon^{\prime}\right)$ (the 0 th coefficient of $x\left(\varepsilon^{\prime}\right)$ ) converges to 0 . Then for any positive $\varepsilon$ we have, for some choice of $\varepsilon^{\prime}$, $\|B x-c x\|<\varepsilon$ and $\left|x_{0}\right|<\varepsilon$. Let $x^{0}=x_{0} e_{0}$ and $x^{1}=x-x^{0}$. Then

$$
\left\|B x^{1}-c x^{1}\right\| \leqq\|B x-c x\|+\left\|B x^{0}-c x^{0}\right\| \leqq \varepsilon+2\|B\| \varepsilon .
$$


We may therefore choose approximating eigenvectors $x(\varepsilon)$ such that $x_{0}$ always vanishes.

For such $x$, let

$$
x^{-}=\sum_{n<0} x_{n} e_{n}, \quad x^{+}=x-x^{-}=\sum_{n>0} x_{n} e_{n} .
$$

Then $B x^{-}$and $c x^{-}$are both orthogonal to $B x^{+}$and $c x^{+}$, so $B x-c x$ is the orthogonal sum of $B x^{-}-c x^{-}$and $B x^{+}-c x^{+}$. Both of the latter have norms therefore less than $\varepsilon$. Either $x^{-}$or $x^{+}$has norm at least $1 / 2$. It follows that approximate eigenvectors can be chosen from either $1_{+}^{2}$ or $1_{-}^{2}$. In the former case $c$ is in $\Pi\left(R^{+}\right)$ where $R^{+}$is the right shift having the positively indexed weights of $B$. By direct comparison of formulae (in terms of $\left.s_{n}\right), i(B)^{+}=i\left(R^{+}\right) \leqq|c| \leqq r\left(R^{+}\right)=r(B)^{+}$, contrary to hypothesis.

In the latter case, $c$ is in $\Pi\left(L^{-}\right)$, where $L^{-}$is the left shift with weights $t_{n}=s_{-n}$. Then $|c| \leqq r\left(L^{-}\right)=r(B)^{-}$, again a contradiction.

So there is a positive number $d$ such that, for some sequence $\varepsilon^{\prime} \rightarrow 0,\left|x_{0}\left(\varepsilon^{\prime}\right)\right| \geqq d$ for all $\varepsilon^{\prime}$.

If $n \geqq N$ then

$$
\left|s_{0} \cdots s_{n-1} x_{0}\right| / c^{n}>(b / c)^{n} d .
$$

Also, using the Cauchy-Schwarz inequality,

$$
\begin{aligned}
\left|x_{n}-\frac{s_{0} \cdots s_{n-1} x_{0}}{c^{n}}\right| \leqq & \left|x_{n}-\frac{s_{n-1} x_{n-1}}{c}\right|+\left|\frac{s_{n-1} x_{n-1}}{c}-\frac{s_{n-1} s_{n-2} x_{n-2}}{c^{2}}\right| \\
& +\cdots+\left|\frac{s_{n-1} \cdots s_{1} x_{1}}{c^{n-1}}-\frac{s_{n-1} \cdots s_{0} x_{0}}{c^{n}}\right| \\
\leqq & \frac{1}{c}\left(\left|c x_{n}-s_{n-1} x_{n-1}\right|^{2}+\cdots+\left|c x_{1}-s_{0} x_{0}\right|^{2}\right)^{1 / 2} \\
& \cdot\left(1+\left|\frac{s_{n-1}}{c}\right|^{2}+\cdots+\left|\frac{s_{n-1} \cdots s_{1}}{c^{n-1}}\right|^{2}\right)^{1 / 2} \\
\leqq & \frac{1}{c}\|B x-c x\|\left(1+\left[\frac{\|B\|}{c}\right]^{2}+\cdots+\left[\frac{\|B\|}{c}\right]^{2(n-1)}\right)^{1 / 2} \\
< & \frac{\varepsilon}{c}\left[\frac{(\|B\| / c)^{2 n}-1}{(\|B\| / c)^{2}-1}\right]^{1 / 2} \cdot(*)
\end{aligned}
$$

Fix $n \geqq N$ such that $(b / c)^{n} d>2$, then choose $\varepsilon>0$ such that $\left(^{*}\right) \leqq 1$. We then have

$$
\left|x_{n}\right| \geqq\left|\frac{s_{0} \cdots s_{n-1} x_{0}}{c^{n}}\right|-\left|\frac{s_{0} \cdots s_{n-1} x_{0}}{c^{n}}-x_{n}\right|>1
$$

which is impossible since $x$ is a unit vector.

Therefore $c$ is not in $\Pi(B)$, and the theorem now follows.

Corollary. Either $\Pi(B)$ or $\Pi\left(B^{*}\right)$ is connected. 
Proof. Suppose $\Pi(B)$ is disconnected. Then $i\left(B^{*}\right)^{+} \leqq r\left(B^{*}\right)^{+}=r(B)^{-}<i(B)^{+}$ $=i\left(B^{*}\right)^{-} \leqq r\left(B^{*}\right)^{-}$so $\Pi\left(B^{*}\right)$ is connected.

THEOREM 4. If $\Pi_{0}(B)$ is nonempty, then $\Pi(B)$ is connected. If $\Pi(B)$ is disconnected, then $\Gamma(B)$ is an annulus whose boundary components are contained in distinct components of $\Pi(B)$.

Proof. If $\Pi_{0}(B)$ is nonempty, then by [3] (or straightforward computation), it is an annulus, centered at 0 , of inner radius $p_{1}(B)=\lim \sup _{n}\left|s_{1} \cdots s_{n}\right|^{1 / n}$ and outer radius $p_{2}(B)=\liminf \operatorname{in}_{n}\left|s_{-1} \cdots s_{-n}\right|^{1 / n}$; by direct substitution in terms of $s_{i}$, and standard inequalities among various limits, we have $i(B)^{+} \leqq p_{1}(B) \leqq p_{2}(B) \leqq r(B)^{-}$; $\Pi(B)$ is then connected by Theorem 3 .

Also, $\Gamma(B)$ is an annulus of inner radius $c_{1}(B)=\lim \sup _{n}\left|s_{-1} \cdots s_{-n}\right|^{1 / n}$ and outer radius $c_{2}(B)=\lim \inf _{n}\left|s_{1} \cdots s_{n}\right|^{1 / n}$ provided that $c_{1}(B) \leqq c_{2}(B)$. If $\Pi(B)$ is disconnected, then by Theorem 3 and standard inequalities among limits, $i(B)^{-} \leqq c_{1}(B) \leqq r(B)^{-}<i(B)^{+} \leqq c_{2}(B) \leqq r(B)^{+}$. The second assertion now follows.

Corollary (Kelley). $\Lambda(B)$ is connected.

Proof. $\Lambda=\Pi \cup \Gamma$.

THEOREM 5. Suppose $\left(\left|s_{n}\right|\right)$ and $\left(\left|s_{-n}\right|\right), n>0$, are almost periodic with approximating periodic means $q^{+}$and $q^{-}$, respectively. If $q^{+} \leqq q^{-}$, then $\Lambda(B)=\Pi(B)$ $=\left\{c: q^{+} \leqq|c| \leqq q^{-}\right\}$. If $q^{-}<q^{+}$, then $\Lambda(B)=\left\{c: q^{-} \leqq|c| \leqq q^{+}\right\}$and

$$
\Pi(B)=\left\{c:|c|=q^{-} \text {or }|c|=q^{+}\right\} .
$$

Proof. By (3), $i(B)^{+}=r(B)^{+}=q^{+}$and $i(B)^{-}=r(B)^{-}=q^{-}$. All assertions now follow from Theorems 3 and 4 .

Corollary. Suppose $a=\lim \left|s_{-n}\right|$ and $b=\lim \left|s_{n}\right|$ as $n \rightarrow+\infty$. If $b \leqq a$ then $\Lambda(B)=\Pi(B)=\{c: b \leqq|c| \leqq a\}$. If $a<b$ then $\Lambda(B)=\{c: a \leqq|c| \leqq b\}$ and

$$
\Pi(B)=\{c:|c|=\text { a or }|c|=b\} .
$$

EXAMPle (Kelley). $s_{n}=1$ for negative $n$, and 2 for positive $n$. Then

$$
\Lambda(B)=\{c: 1 \leqq|c| \leqq 2\} \text { and } \Pi(B)=\{c:|c|=1 \text { or }|c|=2\}
$$

THEOREM 6. $\Pi(L)=\Lambda(L)=\Lambda(R)$.

Proof. The second inequality holds because $L$ and $R$ are adjoint to each other. $\Gamma(L)$, being equal to $\Pi_{0}(R)$, is either empty or $\{0\}$. Since $\Lambda=\Pi \cup \Gamma$, either $\Pi(L)=\Lambda(L)$ or $\Pi(L)=\Lambda(L)-\{0\}$. But the latter case is impossible. For by the corollary (Kelley) to Theorem 1 , either $\Lambda(L)=\{0\}$, in which case $\Pi(L)$ would be empty, or $\Lambda(L)$ is a disk of positive radius, in which case $\Pi(L)$ would fail to be closed. 
LEMMA 7. If $i, c, r$ are any three numbers with $0 \leqq i \leqq c \leqq r$, then there is a positive sequence $\left(s_{n}\right)$ such that

$$
\lim _{n} \inf _{k}\left(s_{k+1} \cdots s_{k+n}\right)^{1 / n}=i, \quad \lim _{n} \sup _{k}\left(s_{k+1} \cdots s_{k+n}\right)^{1 / n}=r,
$$

and

$$
\lim _{n} \inf \left(s_{1} \cdots s_{n}\right)^{1 / n}=c .
$$

If $i, p, c$ are any three numbers with $0 \leqq i \leqq p \leqq r$, then there is a positive sequence $\left(s_{n}\right)$ which satisfies the above equalities for $i$ and $r$, and such that

$$
\lim _{n} \sup \left(s_{1} \cdots s_{n}\right)^{1 / n}=p .
$$

Proof. We construct the sequences and omit the verifications, which consist of routine analysis; details are in [4].

If $i=r$, let $s_{n}=r$ for all $n$ (or positive $s_{n} \rightarrow 0$ if $r=0$ ).

Suppose $i<r$. Choose a monotone nonincreasing sequence of positive numbers $i_{k}$ converging to $i$. (If $i>0$ we may take $i_{k} \equiv i$.) Choose a rational sequence $\left(r_{k}=p_{k} / q_{k}\right), p_{k}, q_{k}$ integers, such that $p_{k}$ and $q_{k}-p_{k}$ tend to infinity and $i_{k}\left(r / i_{k}\right)^{r_{k}}$ converges to $c$. Let $\left(s_{n}\right)$ consist of a sequence of cycles $C_{k}$, where each $C_{k}$ is a sequence of $r$ 's of length $p_{k}$, followed by a sequence of $i_{k}$ 's of length $q_{k}-p_{k}$.

This gives the first required sequence, for $i, c, r$. To obtain the second, for $i, p, r$, proceed as before but let the $i_{k}$ 's precede the $r$ 's in each cycle $C_{k}$.

Note. $\Gamma(R)$ is a disk; let $c(R)$ denote its radius. Define $c_{1}(B), c_{2}(B), p_{1}(B)$, and $p_{2}(B)$ as in the proof of Theorem 4 .

THEOREM 8. If $i, c, r$ are any three numbers with $0 \leqq i \leqq c \leqq r$, then there is an injective right shift $R$ with $i(R)=i, c(R)=c$, and $r(R)=r$. If $i, p_{1}, p_{2}, r$ are any four numbers with $0 \leqq i \leqq p_{1} \leqq p_{2} \leqq r$, then there is an injective two-sided shift $B$ with $i(B)=i, p_{1}(B)=p_{1}, p_{2}(B)=p_{2}$, and $r(B)=r$. If $i^{-}, c_{1}, r^{-}, i^{+}, c_{2}, r^{+}$are any six numbers with $0 \leqq i^{-} \leqq c_{1} \leqq r^{-}<i^{+} \leqq c_{2} \leqq r^{+}$, then there is an injective two-sided shift $B$ with $i^{-}(B)=i^{-}, c_{1}(B)=c_{1}, r^{-}(B)=r^{-}, i^{+}(B)=i^{+}, c_{2}(B)=c_{2}$, and $r^{+}(B)=r^{+}$.

Proof. We exhibit the three asserted shifts by constructing the sequences of weights $\left(s_{n}\right)$, using the two constructions of Lemma 7 in suitable combinations; the verifications are then routine.

(1) Use the first construction (of Lemma 7) directly.

(2) Let $s_{0}=1$. For $n>0$, use the second construction (Lemma 7), with $i=i$, $p=p_{1}$, and $r=r$. Let $s_{-n}=t_{n}$ where $\left(t_{n}\right)$ satisfies the first set of conditions (Lemma 7), with $i=i, c=p_{2}$, and $r=r$.

(3) Let $s_{0}=1$. For $n>0$, use the first construction of Lemma 7, with $i=i^{+}$, $c=c_{2}$, and $r=r^{+}$. Let $s_{-n}=t_{n}$ where $\left(t_{n}\right)$ satisfies the second set of conditions (Lemma 7), with $i=i^{-}, p=c_{1}$, and $r=r^{-}$.

NoTE. In (2), modification, or caution, may be required if $p_{1}=p_{2}$. For $\Pi_{0}(B)$ is 
actually the annulus of convergence of a power series involving the $s_{n}$; here it is either a circle of radius $p_{1}=p_{2}$, or empty. To ensure that it is indeed the circle, we need only proceed with caution in constructing the sequences $\left(s_{n}\right)$ and $\left(t_{n}\right)$.

\section{REFERENCES}

1. R. Gellar, Cyclic vectors and parts of the spectrum of a weighted shift, Univ. of New Mexico, 1968, preprint.

2. P. R. Halmos, A Hilbert space problem book, Van Nostrand, Princeton, N. J., 1967, pp. 38-49.

3. R. L. Kelley, Weighted shifts on Hilbert space, Thesis, Univ. of Michigan, 1966, pp. 19-33.

4. W. C. Ridge, Composition operators, Thesis, Indiana Univ., 1969, pp. 47-69.

INDIANA UNIVERSITY,

BLOOMINGTON, INDIANA 